\title{
ON RANK 4 PROJECTIVE PLANES
}

\author{
O. BACHMANN \\ Département de mathématiques \\ Ecole polytechnique fédérale \\ $\mathrm{CH}-1007$ Lausanne, Suisse \\ (Received October 4, 1979)
}

ABSTRACT. Let a finite projective plane be called rank m plane if it admits a collineation group $G$ of rank $m$, let it be called strong rank $m$ plane if moreover $G_{P}=G_{1}$ for some point-line pair $(P, 1)$. It is well known that every rank 2 plane is desarguesian (Theorem of Ostrom and Wagner). It is conjectured that the only rank 3 plane is the plane of order 2. By [1] and [7] the only strong rank 3 plane is the plane of order 2. In this paper it is proved that no strong rank 4 plane exists.

KEY WORDS AND PHRASES. Projective planes, rank 4 groups. 1980 MATHEMATICS SUBJECT CLASSIFICATION CODE: 50 Geometry.

1. INTRODUCTION.

In [6] Ka1laher gives restrictions for the order $n$ of a finite rank 3 pro- 
jective plane and conjectures that no such plane exists if $n \neq 2$. Let a finite projective plane be called a strong rank m projective plane if it admits a rank m collineation group $G$ such that $G_{P}=G_{1}$ for some point-line pair $(P, 1)$. By Bachmann [1] and Kantor [7] no strong rank 3 projective plane of order $n \neq 2$ exists. If the conjecture is true that for projective designs the representations on the points and on the blocks of an arbitrary transitive collineation group are similar (see Dembowski [2], p. 78), then every rank m projective plane is a strong rank m plane.

We shall prove in this article the following

THEOREM: No strong rank 4 projective plane exists.

To prove the Theorem we first divide the strong rank 4 planes into 3 classes (see Lemma 2 and 3). Then we associate with each such plane $(0,1)$-matrices $A$ and $C$ of trace $O$ (see [3]). Finally we show that for each class the trace condition contradicts the integrality of the multiplicities of the eigenvalues of $A$ or $C$.

We shall use the following notations, definitions and basic results (see Dembowski [2]):

A collineation group of a projective plane has equally many point orbits and line orbits. The rank of a transitive permutation group is the number of orbits of the stabilizer of one of the permuted elements. If $G$ is a (point or line) transitive collineation group of a projective plane, then the point and line ranks are equal (Kantor [8]). A rank m projective plane is a projective plane which admits a transitive collineation group whose (point or line) rank is $\mathrm{m}$ $(m \geq 2)$. The lines (points) are identified with the set of points (lines) on them. We write $P \in 1^{G}$ if and only if $P \in 1^{\gamma}$ for all $\gamma \in G$. 


\section{PROOF OF THE THEOREM.}

Let $\mathbb{P}=(P, L, \epsilon)$ be a projective plane of finite order $n$ and let $G$ be a rank 4 collineation group of $\mathbb{P}$ such that $G_{P_{0}}=G_{1}$ for some point-line pair $\left(P_{0}, 1_{0}\right)$. It is easily seen that $n \geqslant 3$. A bijective map $\sigma: P \longrightarrow L$ is defined by $P^{\sigma}=1$ if and only if $P=P_{0}^{\gamma}$ and $1=1_{0}^{\gamma}$ for some $\gamma \in G$. If $i \in \mathbb{N}$ we write $1_{i}$ for $P_{i}^{\delta}$. Clearly $P_{0}^{\delta}=1_{0}$ and

$$
P^{\sigma \gamma}=P^{\gamma \sigma}, 1^{\sigma^{-1} \gamma}=1^{\gamma \sigma^{-1}} \text { for all } P \in P, 1 \in L, \gamma_{\in} \text {. }
$$

For $P \in P \quad G_{P}$ has exactly 4 orbits $\{P\}, \Delta(P), \quad \Gamma(P), \dot{I}(P)$. We choose the notation in such a way that

$$
(\Delta(P))^{\gamma}=\Delta\left(P^{\gamma}\right),(\Gamma(P))^{\gamma}=\Gamma\left(P^{\gamma}\right),(\pi(P))^{\gamma}=\pi\left(P^{\gamma}\right) \text { for all } P \in P, \gamma \in G(2)
$$

LEMMA 2.1: If $\Lambda_{1}, \Lambda_{2}, \Lambda_{3} \in\{\triangle, \Gamma, \pi\}$, then $\left|\Lambda_{1}(A) \cap \Lambda_{2}(B)\right|=$ $\left|\Lambda_{1}\left(A^{\prime}\right) \cap \Lambda_{2}\left(B^{\prime}\right)\right| \quad$ if $\quad A \in \Lambda_{3}(B)$ and $A^{\prime} \in \Lambda_{3}\left(B^{\prime}\right)$.

PROOF: If $A \in \Lambda_{3}(B), A^{\prime} \in \Lambda_{3}\left(B^{\prime}\right)$, then for some $\gamma \in G, \gamma_{0} \in G_{B}$

$$
\begin{aligned}
& B^{\prime}=B^{\gamma}=B_{0}^{\gamma_{0} \gamma}, A^{\prime}=A^{\gamma_{0} \gamma} \text {, whence by (2) } \\
& \left|\Lambda_{1}\left(A^{\prime}\right) \cap \Lambda_{2}\left(B^{\prime}\right)\right|=\left|\Lambda_{1}(A){ }^{\gamma_{0}} \gamma_{0} \Lambda_{2}(B){ }^{\gamma_{0}} \Upsilon_{1}\right|=\left|\left(\Lambda_{1}(A) \cap \Lambda_{2}(B)\right)^{\Upsilon_{0}} \Upsilon_{1}\right|= \\
& \left|\Lambda_{1}(\mathrm{~A}) \cap \Lambda_{2}(\mathrm{~B})\right| \text {. }
\end{aligned}
$$

LEMMA 2.2: Suppose that $P_{0} \in 1_{0}$. Then $1_{0}-\left\{P_{0}\right\}$ and $P_{0}-\left\{1_{0}\right\}$ are $G_{P_{0}}^{-}$ orbits, say $\Delta\left(P_{0}\right)=1_{0}-\left\{P_{0}\right\}$ and $1_{2} P_{0}=P_{0}-\left\{1_{0}\right\}$ with $P_{2} P_{0}=\Gamma\left(P_{0}\right) . P_{1} \in \Delta\left(P_{0}\right)$ and $P_{3} \in \Pi\left(P_{0}\right)$ can be chosen such that $P_{1} \in 1_{0} ; P_{0}, P_{2}, P_{3} \in 1_{2} ; P_{2} \in 1_{1} ; P_{1} \notin 1_{3}$ (Fig. 1).

The case described by Lemma 2 will be called case I.

PROOF: If $1_{0}-\left\{P_{0}\right\}$ is not a $G_{P_{0}}$ - orbit, then it is the union of 2 orbits, say $1_{0}-\left\{P_{0}\right\}=\Delta\left(P_{0}\right) \cup \Gamma\left(P_{0}\right)$. Then $P_{0}-\left\{1_{0}\right\}$ is a line orbit ${ }^{G} P_{0}$ and $\pi\left(P_{0}\right)=$ $P^{G} P_{0}$ with $1=P^{\sigma}$. This leads to the contradiction 
$\therefore \quad n=\left|{ }_{1}^{G} P_{0}\right|=\left|P^{\sigma}{ }^{G} P_{O}\right|=\left|P^{G} P_{0}{ }^{\sigma}\right|=\left|P^{G} P_{O}\right|=\left|P-1_{0}\right|=n^{2}$.

Hence we may assume that $\Delta\left(P_{0}\right)=1_{0}-\left\{P_{0}\right\}$.

Dually: $P_{0}-\left\{1_{0}\right\}$ is a $G_{P_{0}}$ - orbit, say $1_{2} P_{0}=P_{0}-\left\{1_{0}\right\}$ where $P_{2} P_{0}=\Gamma\left(P_{0}\right)$ (note that $P_{0} \notin 1_{1}$ ).

$$
\left|P_{2}^{G_{P_{O}}}\right|=\left|1_{2}{ }_{P_{0}}\right|=\left|P_{0}-\left\{1_{o}\right\}\right|=n \quad \text { implies } \Gamma\left(P_{0}\right) \cap 1_{2}=\left\{P_{2}\right\} \text {. }
$$

For any point $Q \neq P_{0}, P_{2}$ on $1_{2}$ holds $Q{ }^{P_{0}}=\pi\left(P_{0}\right)$.

Let $P_{1}^{\prime} \in \Delta\left(P_{0}\right)$. If $P_{2} \in 1_{1}^{\prime}$ put $P_{1}=P_{1}^{\prime}$. If $P_{2} \notin 1_{1}^{\prime}$ then

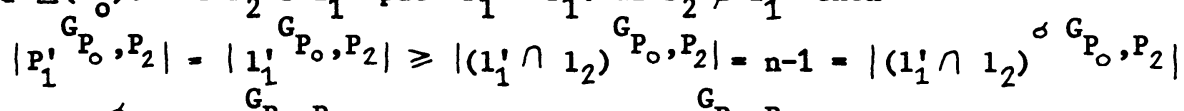

$>\left|\left(\left(1_{1}^{i} \cap 1_{2}\right)^{\sigma} \cap 1_{0}\right){ }^{G_{P_{0}}, P_{2}}\right|$; this implies $P_{1}^{G_{0}}, P_{2}=P_{1}$ for some point

$P_{1} \in \Delta\left(P_{0}\right)$ and hence $P_{2} \in 1_{1}$.

It remains to prove that $P_{3} \in I\left(P_{0}\right) \cap 1_{2}$ exists such that $P_{1} \notin 1_{3}$. If no such $P_{3}$ exists then $P_{1} \in Q^{\sigma}$ for all $Q \in 1_{2}-\left\{P_{0}, P_{2}\right\}$ and hence $G P_{0}, P_{2} \leqslant G_{P_{0}}, P_{1}$. Let $\gamma \in G$ be such that $P_{2}^{\gamma}=P_{0}$. Then $1_{2}^{\gamma}=1_{0}$ and therefore $P_{0}^{\gamma} \in 1_{0}, P_{0}^{\gamma} \neq P_{0}$ and $P_{0}^{\gamma \gamma_{0}}=P_{1}$ for some $\gamma_{0} \in G_{P_{0}}$. It follows that $G_{P_{0}, P_{1}}=\left(\bar{\gamma} r_{0}\right)^{-1} G_{P_{0}, P_{2}} \bar{\gamma} r_{0}$.

Hence

$$
G_{P_{0}, P_{1}}=G_{P_{0}, P_{2}}
$$

Further

$$
P_{2} \notin 1_{1}^{Y_{0}} \text { for some } Y_{0} \in G_{P_{0}}
$$

for otherwise $P_{2}^{\gamma_{0}^{\prime}} \in 1_{1}^{\gamma_{0}^{\prime \prime}}$ for all $\gamma_{0}^{\prime}, \gamma_{0}^{\prime \prime} \in G_{P_{0}}$ which cannot occur.

$$
P_{2}^{\gamma_{0}} \in 1_{1} \text { for some } r_{0} \in G_{P_{0}} \text { if and only if } \gamma_{0} \in G_{P_{2}} \text {. }
$$

To prove (5) note that by (4) through any point of $1_{2}-\left\{P_{0}\right\}$ goes at least one and hence exactly one line of ${ }_{1}{ }_{1} P_{Q} \quad$ (3) and $P_{2} \in 1_{1}$ then imply (5).

Let's apply (5) to $G_{P_{1}}$ in place of $G_{P_{0}}$ : $\Delta\left(P_{1}\right)=1_{1}-\left\{P_{1}\right\} ; \quad \Gamma\left(P_{1}\right)=\Gamma\left(P_{0}^{\gamma}\right)=\left(\Gamma\left(P_{0}\right)\right)^{\gamma}=P_{2}^{G} P_{0}^{\gamma}=P_{0}^{\gamma^{-1} G_{P_{0}}^{\gamma}}=P_{0}^{G} P_{1}$ 
where $\gamma \in G$ such that $P_{0}^{\gamma}=P_{1}, P_{2}^{\gamma}=P_{0} ; \mathbb{I}\left(P_{1}\right)=S^{G} P_{1}$ for some $S \in 1_{0}-$ $\left\{P_{0}, P_{1}\right\}$; hence $P_{0}^{Y_{1}} \in 1_{2}$ for some $\gamma_{1} \in G_{P_{1}}$ if and only if $\gamma_{1} \in G_{P_{0}}$.

It follows that $R \notin P_{0} P_{1}$ for any $R \in 1_{2}-\left\{P_{0}, P_{2}\right\}$. Let $r=R^{\sigma}$ for some such $R$.

Of the 3 orbits $\left(P_{0}, 1_{1}\right)^{G},\left(P_{0}, 1_{2}\right)^{G},\left(P_{0}, r\right)^{G}$ induced by $G$ on $P \times L-$ $\left(P_{0}, 1_{0}\right)^{G}$ only one consists of flags. Thus $\left(P_{1}, 1_{0}\right)$ and $\left(P_{1}, r\right)$ and then also $\left(P_{0}, 1_{1}\right)$ and $\left(R, 1_{1}\right)$ belong to the same G-orbit. This contradicts $R \notin P_{0}{ }^{P_{1}}$ Hence there exists $P_{3} \in \mathbb{H}\left(P_{0}\right) \cap 1_{2}$ such that $P_{1} \notin 1_{3}$.

LEMMA 2.3: Suppose that $P_{0} \notin 1_{0}$. Then $1_{0}$ and dually $P_{0}$ are $G_{P_{0}}$-orbits, say $\Delta\left(P_{0}\right)=1_{0} \cdot P_{1} \in \Delta\left(P_{0}\right), P_{2} \in \Gamma\left(P_{0}\right), P_{3} \in \pi\left(P_{0}\right)$ can be chosen such that either $P_{0}, P_{2}, P_{3} \in 1_{1} ; P_{1} \in 1_{2}, 1_{3} ; P_{2} \notin 1_{3} ; P_{3} \notin 1_{2}$ or $P_{0}, P_{1}, P_{3} \in 1_{2} ; P_{1} \in 1_{0} ; I\left(P_{0}\right) \cap 1_{2}=\left\{P_{2}^{\gamma_{0}}\right\}$ for some $\gamma_{0} \in G_{P_{0}} ; P_{2}^{\gamma_{0}} \in 1_{1} ; P_{1}, P_{2}$, $\mathrm{P}_{3} \notin 1_{1}, 1_{3}$. In both cases $\mathrm{n} \geqslant 4$.

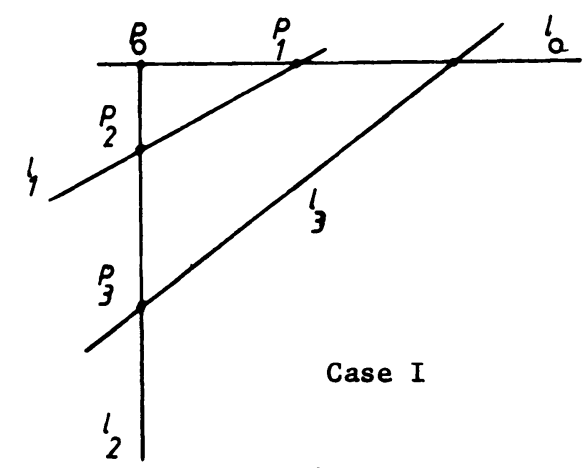

Figure 1

The 2 cases described by Lemma 3 will be called case III resp. case II2 (Fig. 2).

PROOF: It is easily seen that $1_{0}$ and $P_{0}$ are $G_{P_{0}}$ - orbits; say $\Delta\left(P_{0}\right)=1_{0}$. Let $P_{1} \in \Delta\left(P_{0}\right)$. We have to distinguish 2 cases:

Case III: $P_{0} \in 1_{1}$

Case II2: $P_{0} \notin 1_{1}$.

CASE IIl: Clearly $P_{0}=1_{1}{ }^{G} P_{0}$ and $T\left(P_{0}\right)=P_{2}{ }^{P_{0}}, \quad \pi\left(P_{0}\right)=P_{3}{ }^{P_{0}}$ for some $P_{2}, P_{3} \in 1_{1}-\left\{P_{0}, 1_{0} \cap 1_{1}\right\}$. If $P_{2} \in 1_{3}$ then $\left(P_{2}, 1_{3}\right) \in\left(P_{0}, 1_{1}\right)^{G}$, hence $\left(P_{3}, 1_{2}\right) \in\left(P_{1}, 1_{0}\right)^{G}$, so $P_{3} \in 1_{2}$. 

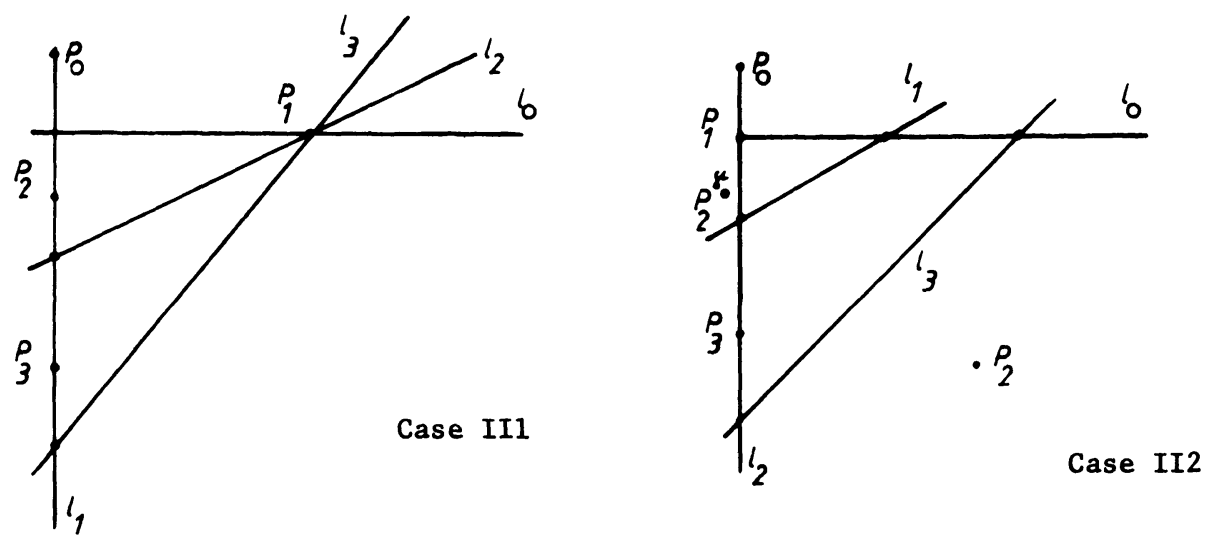

Figure 2

Analogously $P_{2} \in 1_{3}$ if $P_{3} \in 1_{2}$. Thus

$$
P_{2} \in 1_{3} \text { if and only if } P_{3} \in 1_{2} .
$$

Similarly one proves

$$
P_{1} \in 1_{2}, 1_{3} .
$$

If $n>3$ then, by (6), we can choose $P_{2}, P_{3}$ such that $P_{2} \notin 1_{3}, P_{3} \notin 1_{2}$. Let's show that $n>3$ (Fig. 3). Suppose that $n=3$. Put $P_{4}=1_{0} \cap 1_{1}$.

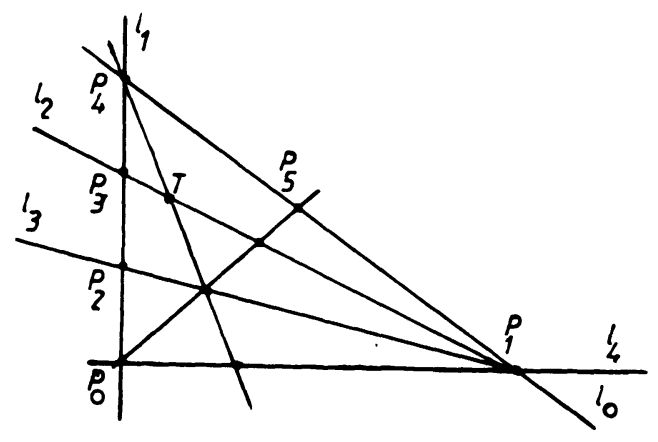

Figure 3
Then, since $P_{0} \in I_{1}$ and $P_{1} \in I_{0}$, $1_{4}=P_{0} P_{1}$. Let $P_{5} \in 1_{0}-\left\{P_{1}, P_{4}\right\}$. Then $P_{0} \in 1_{5}$ and then $1_{5} \cap 1_{2}=$ $\left(P_{\circ} P_{5} \cap 1_{3}\right) P_{4} \cap 1_{2} \cdot$ Denote this point by T. Clearly $\mathrm{P}_{2} \mathrm{P}_{5} \cap \mathrm{l}_{2}=$ T. Since $\left(\mathrm{P}_{2} \mathrm{P}_{5}\right)^{\delta-1} \in 1_{2} \cap 1_{5}$ we obtain the contradiction $\left(\mathrm{P}_{2} \mathrm{P}_{5}\right)^{\delta-1} \in \mathrm{P}_{2} \mathrm{P}_{5}$. 
CASE II 2: We may assume that $P_{0}, P_{1}, P_{3} \in 1_{2}$ where $P_{2} \in \Gamma\left(P_{0}\right)$. Then $G_{P_{0}, P_{1}}=G_{P_{0}, P_{2}}$. We first assume that $n>3 .\left|P_{2}^{G_{P}}\right|=\left|1_{2}{ }^{G_{0}}\right|=n+1$, hence $\left|1_{2} \cap \Gamma\left(P_{0}\right)\right|=1$; let $1_{2} \cap \Gamma\left(P_{0}\right)=\left\{P_{2}^{\gamma_{0}}\right\}$ with some, $\gamma_{0} \in G_{P_{0}}$. Then $P_{3}{ }^{G_{P}, P_{1}}=$ $1_{2}-\left\{P_{0}, P_{1}, P_{2}^{\gamma_{0}}\right\}$ and hence, since $P_{2}^{Y_{0}}$ is invariant under $G_{P_{0}}, P_{1}$ and since $\mathrm{n}>3,1_{1} \cap 1_{2}=\mathrm{P}_{2}^{\Upsilon_{0}}$.

The only G-orbit of $P, x, L$ consisting of flags is $\left(P_{0}, 1_{2}\right)^{G}$. Hence $\left(P_{1}, 1_{2}\right)$, $\left(P_{3}, 1_{2}\right),\left(P_{1}, 1_{0}\right) \in\left(P_{0}, 1_{2}\right)^{G} . P_{0} \notin 1_{1}$ then implies that $\left(P_{2}, 1_{1}\right),\left(P_{2}, 1_{3}\right)$, $\left(P_{0}, 1_{1}\right),\left(P_{2}, 1_{0}\right) \notin\left(P_{0}, 1_{2}\right)^{G}$, in particular $P_{2} \notin 1_{0}, 1_{1}, 1_{3}$.

If $P_{1} \in 1_{3}$ then $\left(P_{1}, 1_{3}\right) \in\left(P_{0}, 1_{2}\right)^{G}$ and hence $\left(P_{3}, 1_{1}\right) \in\left(P_{2}, 1_{0}\right)^{G}$. Since also $\left(P_{0}, 1_{1}\right) \in\left(P_{2}, 1_{0}\right)^{G}$ we have $P_{0}^{\gamma_{1}}=P_{3}$ for some $r_{1} \in G_{1}=G_{P_{1}}$. This implies that $G_{P_{1}}$ is transitive on $1_{2}-\left\{P_{1}, P_{2}^{\gamma_{0}}\right\}$ which is impossible. Hence $P_{1} \notin 1_{3}$.

If $n=3$ then $1_{2}=\left\{P_{0}, P_{1}, P_{2}^{\gamma_{0}}, P_{3}\right\} \cdot \gamma_{0}$ is of order 4 , for if $\gamma_{0}^{2}=1$ then $\left(\mathrm{P}_{2}, 1_{2}^{\gamma_{0}}\right) \in\left(\mathrm{P}_{2}^{\gamma_{0}}, 1_{2}\right){ }^{\mathrm{G}_{\mathrm{P}}}$ which is impossible. Moreover $\mathrm{P}_{2}^{\gamma_{\mathrm{O}}^{2}} \neq \mathrm{P}_{2}$ since otherwisw $\gamma_{0}^{2} \in G_{P_{0}}, P_{2}=1$. It follows that $\left|\left(P_{2} P_{2}^{\gamma_{0}^{2}}\right){ }_{P_{0}}\right|=4$ which contradicts $\left(\mathrm{P}_{2} \mathrm{P}_{2}^{\mathrm{Y}_{\mathrm{O}}^{2}}\right)^{\mathrm{G}_{\mathrm{O}}}=\left\{\mathrm{P}_{2} \mathrm{P}_{2}^{\mathrm{P}_{0}}, \mathrm{P}_{2}^{\Upsilon_{\mathrm{O}} \mathrm{P}_{2}^{\gamma_{\mathrm{O}}}}\right\}$. This completes the proof of the Lemma.

Let us now associate with $(G, \mathbb{P}) 3(0,1)$-matrices.

If $P(P)$ is a G-orbit then let $P^{\prime}(P)$ denote the paired orbit (see Wielandt [9] ). If $Q \in P(P)$ then $: Q=P^{\gamma}$ for some $\gamma \in G$ and $Q^{\gamma} \in(P(P))^{\gamma}=P\left(P^{\gamma}\right)=P(Q)$. Hence $Q^{Y^{-1}}=P \in \rho^{\prime}(Q)$, i.e. 
This implies that in

Case I:

$\Delta^{\prime}(\mathrm{P})=\Gamma(\mathrm{P})$

$\Gamma^{\prime}(\mathrm{P})=\Delta(\mathrm{P})$

$\Pi^{\prime}(P)=\Pi(P)$
Case II1:

$\Delta^{\prime}(\mathrm{P})=\Delta(\mathrm{P})$

$\Gamma^{\prime}(P)=\Pi(P)$ resp. $\Gamma(P)$

$\Pi^{\prime}(\mathrm{P})=\Gamma(\mathrm{P})$ resp. $\Pi(\mathrm{P})$
Case II2:

$\Delta^{\prime}(\mathrm{P})=\Gamma(\mathrm{P})$

$\Gamma^{n}(\mathrm{P})=\Delta(\mathrm{P})$

$\Pi^{\prime}(\mathrm{P})=\Pi(\mathrm{P})$

Now let $P=\left\{P_{1}, P_{2}, \ldots, P_{v}\right\}, L=\left\{1_{1}, 1_{2}, \ldots, 1_{v}\right\}, 1_{k}=P_{k}^{\sigma} \quad(k=1,2$, $\ldots, v)$. Let $A$ be the $(0,1)$-matrix with rows enumerated by the points $P_{k}$ and columns by $\Delta\left(P_{k}\right)$ and such that $\left(P_{k}, \Delta\left(P_{i}\right)\right)=1$ if and only if $P_{k} \in \Delta\left(P_{i}\right)$. Let $B, C$ be the analogous matrices with $\Gamma\left(P_{k}\right)$ resp. $\mathbb{I}\left(P_{k}\right)$ in place of $\Delta\left(P_{k}\right)$.

We have in

case I:

case II1:

case II2:

$$
\begin{aligned}
& A^{t=B, C^{t}=C \quad A^{t}=A, B^{t}=C} \quad \text { if } \Gamma^{\prime}(P)=\Pi(P) \quad A^{t}=B, C^{t}=C \\
& A^{t}=A, B^{t}=B, C^{t}=C \text { if } \Gamma^{\prime}(P)=\Gamma(P) \\
& \text { Let } k=|\Delta(P)|, 1=|\Gamma(P)|, m=|\Pi(P)|, \\
& |\Delta(P) \cap \Delta(Q)|=\left\{\begin{array}{l}
\lambda \\
\mu \\
\nu
\end{array}\right\} \text { if } Q \in\left\{\begin{array}{l}
\Delta(P) \\
\Gamma(P) \\
\Pi(P)
\end{array}\right. \\
& |\Pi(P) \cap \Pi(Q)|=\left\{\begin{array}{l}
\lambda^{\prime} \\
\mu^{\prime} \\
\nu^{\prime}
\end{array}\right\} \text { if } Q \in\left\{\begin{array}{l}
\Pi(P) \\
\Delta(P) . \\
\Gamma(P)
\end{array}\right.
\end{aligned}
$$

A straightforward calculation shows that

$$
\begin{aligned}
I+A+B+C & =J, \text { the vxv-matrix with } I^{\prime} \text { s in every entry } \\
A^{t} A & =k I+\lambda A+\mu B+\nu C \\
C^{t} C & =m I+\mu^{\prime} A+\nu^{\prime} B+\lambda^{\prime} C
\end{aligned}
$$



A $J=k J$
B $\mathrm{J}=1 \mathrm{~J}$
$\mathrm{C} \mathrm{J}=\mathrm{m} \mathrm{J}$.

Now we determine the eigenvalues of $A$ in case III and of $C$ in the cases I and II2.

$$
\begin{aligned}
& \text { CASE II1: . } \quad k=n+1 \\
& 1=n_{2}(n+1) \quad \text { where } n_{2}=\left|P_{2}{ }^{G_{0}, P_{1}}\right| \\
& m=n_{3}(n+1) \quad \text { where } n_{3}=\left|P_{3} P_{0}, P_{1}\right| \\
& \mathrm{k}+1+\mathrm{m}+1=\mathrm{v}=\mathrm{n}^{2}+\mathrm{n}+1, \mathrm{n}_{2}+\mathrm{n}_{3}=\mathrm{n}-1, \lambda=\mu=\nu=1 \text {. }
\end{aligned}
$$

It follows that $A^{2}=A^{t} A=(n+1) I+A+B+C=n I+J$; hence $(A-(n+1) I)\left(A^{2}-n I\right)=0$. This gives the eigenvalues of $A$ :

$$
\begin{array}{ll}
\lambda_{1}=n+1, \lambda_{2,3}= \pm \sqrt{n} . \\
\text { CASE I: } \quad k=1=n, m=n(n-1), k+1+m+1=v=n^{2}+n+1 .
\end{array}
$$

We have

$$
\begin{aligned}
& \lambda^{\prime}=\left|\pi\left(\mathrm{P}_{0}\right) \cap \boldsymbol{I}\left(\mathrm{P}_{3}\right)\right| \\
& \mu^{\prime}=\left|\mathbb{I}\left(\mathrm{P}_{0}\right) \cap \mathbb{I}\left(\mathrm{P}_{1}\right)\right| \\
& \nu^{\prime}=\left|\mathbb{I}\left(\mathrm{P}_{0}\right) \cap \mathbb{I}\left(\mathrm{P}_{2}\right)\right| .
\end{aligned}
$$

Let's calculate $\lambda$ ':

$$
n(n-1)=\left|\Pi\left(P_{3}\right)\right|=\left|\Pi\left(P_{3}\right) \cap \Delta\left(P_{0}\right)\right|+\left|\pi\left(P_{3}\right) \cap \Gamma\left(P_{0}\right)\right|+\left|\Pi\left(P_{3}\right) \cap \pi\left(P_{0}\right)\right|+1
$$

(note that $\Gamma\left(P_{3}\right)=P_{2}^{G_{3}}$ and hence $P_{0} \in \Pi\left(P_{3}\right)$ ).

$$
n=\left|\Delta\left(P_{0}\right)\right|=\left|\Delta\left(P_{0}\right) \cap \Delta\left(P_{3}\right)\right|+\left|\Delta\left(P_{0}\right) \cap \Gamma\left(P_{3}\right)\right|+\left|\Delta\left(P_{0}\right) \cap \Pi\left(P_{3}\right)\right| \text {. }
$$

Clearly

$$
\begin{aligned}
& \left|\Delta\left(P_{0}\right) \cap \Delta\left(P_{3}\right)\right|=1 \\
& \left|\Delta\left(P_{0}\right) \cap \Gamma\left(P_{3}\right)\right|=\left|\Delta\left(P_{3}\right) \cap \Gamma\left(P_{0}\right)\right|=2 .
\end{aligned}
$$


PROOF of (12): $P_{0} \in \mathbb{H}\left(P_{3}\right)$ and $P_{3} \in \Pi\left(P_{0}\right)$, hence, by Lemma $1, \mid \Delta\left(P_{0}\right) \cap \Gamma\left(P_{3} \mid\right.$ $=\left|\Delta\left(P_{3}\right) \cap \Gamma\left(P_{0}\right)\right| \cdot P_{2} \notin 1_{1}^{\gamma_{0}}$ for some $\gamma_{0} \in G_{P}$. Thus $\left|P_{3} P_{0}, P_{2}\right|=n-1$ implies

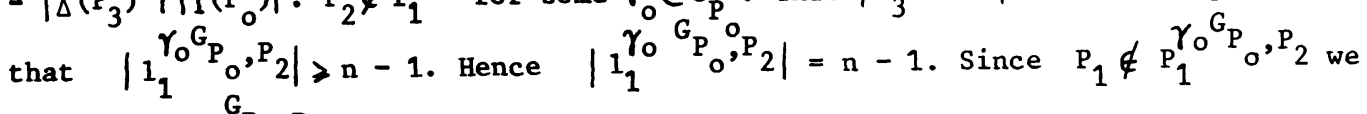
then have $P_{1}^{G} P_{0}, P_{2}=P_{1}$, i.e. $G_{P_{0}, P_{2}} \leqslant G_{P_{0}, P_{1}}$. Since both groups are conjugate

(see the proof of Lemma 2) this gives $G_{P_{0}, P_{1}}=G_{P_{0}, P_{2}} \gamma^{\prime} \gamma^{\prime \prime} \gamma_{0}^{\prime} \gamma_{0}^{\prime \prime}$
Moreover $P_{2} \in 1_{1}^{\gamma_{0}^{*}}$ if and only if $\gamma_{0}^{*} \in G_{P_{1}}$. Thus $P_{2} \in 1_{3}^{0}, P_{2}{ }^{\circ} \in 1_{3}, P_{2} \neq$ $P_{2}$ for some $\gamma_{0}^{\prime}, \gamma_{0}^{\prime \prime} \in G_{P_{0}}$. Since, by the above, $G_{P_{0}, P_{2}}$ is transitive on $1_{0}$ -

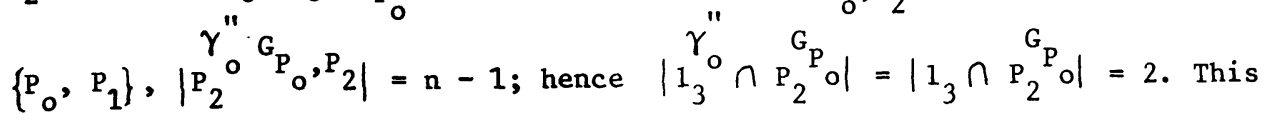
proves (12).

Equations (10), (11), (12) imply

$$
\left|\Pi\left(P_{3}\right) \cap \Delta\left(P_{0}\right)\right|=n-3 .
$$

To determine $\left|\Pi\left(P_{3}\right) \cap \Gamma\left(P_{0}\right)\right|$ we use

$\mathrm{n}=\left|\Gamma\left(\mathrm{P}_{0}\right)\right|=\left|\Gamma\left(\mathrm{P}_{0}\right) \cap \Delta\left(\mathrm{P}_{3}\right)\right|+\left|\Gamma\left(\mathrm{P}_{0}\right) \cap \Gamma\left(\mathrm{P}_{3}\right)\right|+\left|\Gamma\left(\mathrm{P}_{0}\right) \cap \pi\left(\mathrm{P}_{3}\right)\right| \cdot$

By (12) $\left|\Gamma\left(P_{G_{P}}\right) \cap \Delta\left(P_{3}\right)\right|=2$. Since $\Gamma\left(P_{3}\right)={ }_{G_{2}}^{G} P_{3},\left|\Gamma\left(P_{0}\right) \cap \Gamma\left(P_{3}\right)\right|=$ $\left|{ }^{P_{2}}{ }^{G} \circ \cap{ }^{P_{2}}{ }^{P_{3}}\right|=\left|1_{2}{ }^{G} \circ \cap 1_{2}{ }^{{ }^{3}} 3\right|=1$. It follows that

$$
\left|\pi\left(P_{3}\right) \cap \Gamma\left(P_{0}\right)\right|=n-3 .
$$

Equations (9), (13) and (15) imply that

$$
\lambda^{\prime}=n^{2}-3 n+5 .
$$

Analogously we calculate $\mu^{\prime}$ and $\nu^{\prime}$ :

$$
n(n-1)=\left|\Pi\left(P_{1}\right)\right|=\left|\Pi\left(P_{1}\right) \cap \Delta\left(P_{0}\right)\right|+\left|\Pi\left(P_{1}\right) \cap \Gamma\left(P_{0}\right)\right|+\left|\Pi\left(P_{1}\right) \cap \Pi\left(P_{0}\right)\right|
$$

with $\left|\Pi\left(P_{1}\right) \cap \Delta\left(P_{0}\right)\right|=n-1$.

$$
\text { In } \mathrm{n}=\left|\Gamma\left(\mathrm{P}_{0}\right)\right|=\left|\Gamma\left(\mathrm{P}_{0}\right) \cap \Delta\left(\mathrm{P}_{1}\right)\right|+\left|\Gamma\left(\mathrm{P}_{0}\right) \cap \Gamma\left(\mathrm{P}_{1}\right)\right|+\left|\Gamma\left(\mathrm{P}_{0}\right) \cap \mathbb{I}\left(\mathrm{P}_{1}\right)\right|
$$


$\left|\Gamma\left(P_{0}\right) \cap \Delta\left(P_{1}\right)\right|=1$ by the proof of (12) and $\left|\Gamma\left(P_{0}\right) \cap \Gamma\left(P_{1}\right)\right|=\left|P_{2}^{G}{ }^{P_{0}} \cap P_{0}^{G}{ }^{G}\right|=$ $\left|1_{2}{ }^{G} \circ \cap 1_{0}^{G} P_{1}\right|=0$. Hence $\left|\Pi\left(P_{1}\right) \cap \Gamma\left(P_{0}\right)\right|=n-1$ and thus

$$
\mu^{\prime}=(n-1)(n-2) \text {. }
$$

$$
\begin{aligned}
& n(n-1)=\left|\Pi\left(P_{2}\right)\right|=\left|\Pi\left(P_{2}\right) \cap \Delta\left(P_{0}\right)\right|+\left|\Pi\left(P_{2}\right) \cap \Gamma\left(P_{0}\right)\right|+\left|\Pi\left(P_{2}\right) \cap \Pi\left(P_{0}\right)\right| \cdot \\
& \text { In } n=\left|\Delta\left(P_{0}\right)\right|=\left|\Delta\left(P_{0}\right) \cap \Delta\left(P_{2}\right)\right|+\left|\Delta\left(P_{0}\right) \cap \Gamma\left(P_{2}\right)\right|+\left|\Delta\left(P_{0}\right) \cap \Pi\left(P_{2}\right)\right|
\end{aligned}
$$

$\left|\Delta\left(P_{0}\right) \cap \Delta\left(P_{2}\right)\right|=0$ and $\left|\Delta\left(P_{0}\right) \cap \Gamma\left(P_{2}\right)\right|=\left|P_{1}{ }^{G} \circ \cap P_{1}{ }^{G} P_{2}\right|=\left|1_{1}{ }^{G} \circ \cap 1_{1}{ }^{G} P_{2}\right|=1$ (note that $\left|1_{1}{ }^{G}{ }_{2}\right|=\left|P_{1}{ }^{G}{ }_{2}\right|=n$ and hence $\Gamma\left(P_{2}\right)=P_{1}{ }^{P_{2}}$ ). Hence $\left|\Pi\left(P_{2}\right) \cap \Delta\left(P_{0}\right)\right|$ $=n-1$.

Further $\left|\Pi\left(\mathrm{P}_{2}\right) \cap \Gamma\left(\mathrm{P}_{0}\right)\right|=\left|\Gamma\left(\mathrm{P}_{0}\right)\right|-\left|\Gamma\left(\mathrm{P}_{0}\right) \cap \Delta\left(\mathrm{P}_{2}\right)\right|-\left|\Gamma\left(\mathrm{P}_{0}\right) \cap \Gamma\left(\mathrm{P}_{2}\right)\right|-1$ where $\left|\Gamma\left(\mathrm{P}_{\mathrm{o}}\right)\right|=\mathrm{n},\left|\Gamma\left(\mathrm{P}_{\mathrm{o}}\right) \cap \Delta\left(\mathrm{P}_{2}\right)\right|=0$ and $\left|\Gamma\left(\mathrm{P}_{\mathrm{o}}\right) \cap \Gamma\left(\mathrm{P}_{2}\right)\right|=\left|\mathrm{P}_{2}{ }^{\circ} \circ \cap \cap \mathrm{P}_{1} \mathrm{P}_{2}\right|=$ $\left|1_{2}{ }^{G} \circ \cap 1_{1}{ }_{P}{ }^{o}\right|=0$. Hence $\left|\Pi\left(P_{2}\right) \cap \Gamma\left(P_{0}\right)\right|=n-1$. It follows that

$$
v^{\prime}=(n-1)(n-2) \text {. }
$$

Equations (16), (17) and (18) imply that $c^{2}=C^{t} C=n(n-1) I+$ $(n-1)(n-2)(A+B)+\left(n^{2}-3 n+5\right) C=n(n-1) I+(n-1)(n-2)(J-I)+3 C$ and then $(C-n(n-1) I)\left(C^{2}-3 C-2(n-1) I\right)=0$.

The eigenvalues of $c$ are $\lambda_{1}=n(n-1) ; \lambda_{2,3}=(3 \pm \sqrt{8 n+1}) / 2$.

CASE II2: $k=1=n+1, m=(n-2)(n+1), k+1+m+1=v=n^{2}+n+1$. By the proof of Lemma $3 n \geqslant 4$. Let's determine $\lambda^{\prime}, \mu^{\prime}, \nu^{\prime}$ :

$$
\begin{gathered}
(\mathrm{n}+1)(\mathrm{n}-2)=\left|\pi\left(\mathrm{P}_{3}\right)\right|=\left|\mathbb{I}\left(\mathrm{P}_{3}\right) \cap \Delta\left(\mathrm{P}_{0}\right)\right|+\left|\mathbb{I}\left(\mathrm{P}_{3}\right) \cap \Gamma\left(\mathrm{P}_{0}\right)\right|+\mid \pi\left(\mathrm{P}_{3}\right) \cap \\
\mathbb{I}\left(\mathrm{P}_{0}\right) \mid-1 . \text { In } \mathrm{n}+1=\left|\Delta\left(\mathrm{P}_{0}\right)\right|=\left|\Delta\left(\mathrm{P}_{0}\right) \cap \Delta\left(\mathrm{P}_{3}\right)\right|+\left|\Delta\left(\mathrm{P}_{0}\right) \cap \Gamma\left(\mathrm{P}_{3}\right)\right|+\mid \Delta\left(\mathrm{P}_{0}\right) \cap \\
\mathbb{I}\left(\mathrm{P}_{3}\right) \mid \text { clearly }\left|\Delta\left(\mathrm{P}_{0}\right) \cap \Delta\left(\mathrm{P}_{3}\right)\right|=1 \text {. Let's show that } \\
\left|\Delta\left(\mathrm{P}_{0}\right) \cap \Gamma\left(\mathrm{P}_{3}\right)\right|=\left|\Delta\left(\mathrm{P}_{3}\right) \cap \Gamma\left(\mathrm{P}_{0}\right)\right|=2 \\
\left|\Delta\left(\mathrm{P}_{1}\right) \cap \Gamma\left(\mathrm{P}_{0}\right)\right|=2 .
\end{gathered}
$$


PROOF of (19) and (20): By Lemma $1\left|\Delta\left(P_{0}\right) \cap \Gamma\left(P_{3}\right)\right|=\left|\Delta\left(P_{3}\right) \cap \Gamma\left(P_{0}\right)\right|$. For $\gamma_{0}^{\prime} \in G_{P_{0}}$

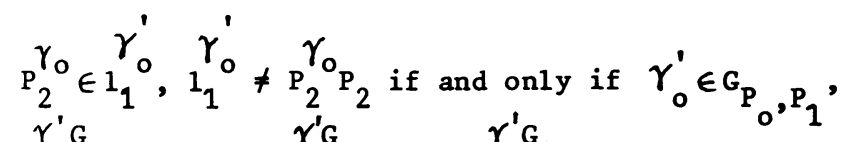

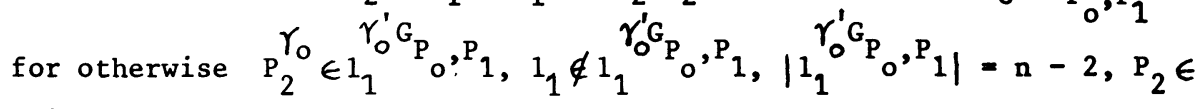

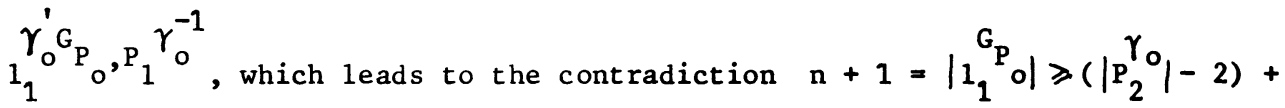
$\left(\left|P_{2}\right|-2\right)=2(n-1)$.

Further

$$
{ }_{\mathrm{P}_{2}}^{\bar{\gamma}_{\mathrm{o}}} \notin 1_{1} \cup \mathrm{P}_{2}^{\gamma_{\mathrm{O}_{2}}} \text { for some } \bar{\gamma}_{\mathrm{o}} \in \mathrm{G}_{\mathrm{P}_{\mathrm{o}}} \text { : }
$$

otherwise, since $\mid P_{2}{ }_{P}$ o $\mid \geqslant 5$, every line of ${ }_{1}{ }_{1}{ }_{0}$ o would contain at least 3 points of $\mathrm{P}_{2}{ }^{\mathrm{G}} \mathrm{O}$ and this would imply that a point of $\mathrm{P}_{2} \mathrm{P}_{\mathrm{O}}-\left(1_{1} \cup \mathrm{P}_{2}{ }^{\gamma_{\mathrm{O}}} \mathrm{P}_{2}\right)$ exists. By (21)

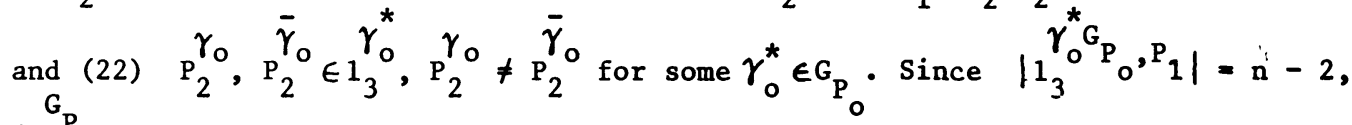
$\mid P_{2}{ }^{G}$ on $1_{3}|=| \Gamma\left(P_{0}\right) \cap \Delta\left(P_{3}\right) \mid=2$. This proves (19).

Each of the $n-2$ lines of ${ }_{1}{ }_{3}{ }{ }_{0}-\left\{P_{2}^{\gamma_{\circ}}{ }_{P_{2}}\right\}$ through ${ }_{2} \gamma_{0}$ contains exactly one point of $\mathrm{P}_{2}^{\mathrm{F}_{\mathrm{P}}} \mathrm{o}-\left\{\mathrm{P}_{2}^{\gamma_{o}}\right\}$. Together with $\mathrm{P}_{2}^{\gamma_{0}}, \mathrm{P}_{2}$ this gives $n$ points of $\mathrm{P}_{2}{ }_{\mathrm{P}}$. It follows that exactly one point of $P_{2}{ }^{P_{0}}-\left\{P_{2}^{\gamma_{\circ}}\right\}$ lies on $1_{1}$. This proves (20).

By means of (19) we obtain $\left|\mathbb{I}\left(\mathrm{P}_{3}\right) \cap \Delta\left(\mathrm{P}_{0}\right)\right|=\mathrm{n}-2$.

In $n+1=\left|\Gamma\left(P_{0}\right)\right|=\left|\Gamma\left(P_{0}\right) \cap \Delta\left(P_{3}\right)\right|+\left|\Gamma\left(P_{0}\right) \cap \Gamma\left(P_{3}\right)\right|+\left|\Gamma\left(P_{0}\right) \cap \pi\left(P_{3}\right)\right|$ $\left|\Gamma\left(P_{0}\right) \cap \Delta\left(P_{3}\right)\right|=2$ by (19) and $\left|T\left(P_{0}\right) \cap T\left(P_{3}\right)\right|=\left|P_{2}{ }^{G} \circ \cap P_{2}{ }^{G}{ }_{3}\right|=\left|1_{2}{ }^{G} \circ \cap 1_{2}{ }^{{ }}{ }_{3}\right|$ $=1$. Hence $\left|\Pi\left(P_{3}\right) \cap \Gamma\left(P_{0}\right)\right|=n-2$. It follows that $\lambda^{\prime}=n^{2}-3 n+1$. $\mu^{\prime}=\left|\Pi\left(P_{0}\right) \cap \pi\left(P_{1}\right)\right|=\left|\Pi\left(P_{1}\right)\right|-\left|\Pi\left(P_{1}\right) \cap \Delta\left(P_{0}\right)\right|-\left|\Pi\left(P_{1}\right) \cap \Gamma\left(P_{0}\right)\right|$ where $\left|\Pi\left(P_{1}\right)\right|=(n+1)(n-2),\left|\Pi\left(P_{1}\right) \cap \Delta\left(P_{0}\right)\right|=n-2$ and $\left|\Pi\left(P_{1}\right) \cap \Gamma\left(P_{0}\right)\right|=\left|\Gamma\left(P_{0}\right)\right|-$ $\left|\Gamma\left(P_{0}\right) \cap \Delta\left(P_{1}\right)\right|-\left|\Gamma\left(P_{0}\right) \cap \Gamma\left(P_{1}\right)\right| \cdot\left|\Gamma\left(P_{0}\right) \cap \Delta\left(P_{1}\right)\right|=2$ by (20) and $\left|\Gamma\left(P_{0}\right) \cap \Gamma\left(P_{1}\right)\right|=$ $\left|P_{2}{ }^{P_{0}} \cap \cap P_{2}^{{ }^{G} P_{1}}\right|=\left|1_{2}{ }^{G} \circ \cap 1_{2}{ }^{P_{1}}\right|=1$. Hence $\left|\Pi\left(P_{1}\right) \cap \Gamma\left(P_{0}\right)\right|=n-2$ and $\mu^{\prime}=$ 


$$
\begin{aligned}
& (n-2)(n-1) \\
& \nu^{\prime}=\left|\pi\left(\mathrm{P}_{0}\right) \cap \pi\left(\mathrm{P}_{2}\right)\right|=\left|\pi\left(\mathrm{P}_{2}\right)\right|-\left|\Pi\left(\mathrm{P}_{2}\right) \cap \Delta\left(\mathrm{P}_{0}\right)\right|-\left|\Pi\left(\mathrm{P}_{2}\right) \cap \Gamma\left(\mathrm{P}_{0}\right)\right| \text { where } \\
& \left|\pi\left(P_{2}\right)\right|=(n+1)(n-2) \text {, } \\
& \left|\Pi\left(\mathrm{P}_{2}\right) \cap \Delta\left(\mathrm{P}_{0}\right)\right|=\left|\Pi\left(\mathrm{P}_{0}\right) \cap \Delta\left(\mathrm{P}_{1}\right)\right| \text { by Lemma } 1 \\
& =\left|\Delta\left(P_{1}\right)\right|-\left|\Delta\left(P_{1}\right) \cap \Delta\left(P_{0}\right)\right|-\left|\Delta\left(P_{1}\right) \cap \Gamma\left(P_{0}\right)\right| \\
& =(n+1)-1-2=n-2 \text { by (20), } \\
& \left|\Pi\left(P_{2}\right) \cap \Gamma\left(P_{0}\right)\right|=\left|\Pi\left(P_{0}\right) \cap \Gamma_{G}\left(P_{1}\right)\right| \text { by Lemma } 1 \\
& =\left|P_{3}^{G_{P}} \circ \cap P_{0}^{{ }_{0} P_{1}}\right|=\left|\left\{1_{3}^{\gamma_{0}}: \gamma_{0} \in G_{G_{0}}, P_{1} \in 1_{3}^{\gamma_{o}}\right\}\right|=n-2 \text { since }
\end{aligned}
$$

through any point on $1_{0}$ goes exactly one line of $1_{1}{ }_{0}^{\circ}$ and one of $1_{2}{ }_{P}$. Hence $\nu^{\prime}=(n-2)(n-1)$.

It follows that $C^{2}=C^{t} c=(n+1)(n-2) I+(n-1)(n-2)(A+B)+$ $\left(n^{2}-3 n+1\right) C=(n+1)(n-2) I+\left(n^{2}-3 n+2\right)(A+B+C)-C$ and $C^{2}+C-$ $2(n-2) I=(n-1)(n-2) J$ whence $(C-(n+1)(n-2) I)\left(C^{2}+C-2(n-2) I\right)$

=0. The eigenvalues of $C$ are $\lambda_{1}=(n+1)(n-2), \lambda_{2,3}=(-1 \pm \sqrt{8 n-15}) / 2$.

REMARK: Let $\Phi: G \rightarrow \mathrm{GL}_{\mathrm{v}}(\mathbb{C})$ be the matrix representation of $\mathrm{G}$ obtained by associating with each $\gamma \in G$ the corresponding permutation matrix $\Phi(\gamma)$ (the ordering of $P$ is the same as used in constructing the matrices $A, B, C)$. By (2) $\Phi(\gamma)$ commutes with A, B, C for all $\gamma \in G$. Hence, by [9] Theorem $28.4,\{I, A, B, C\}$ is the basis of the commuting algebra $\mathbb{V}(G)$ of $\Phi$. By [9] Theorem $29.5 \mathbb{V}(G)$ is commutative and hence, by [9] Theorem 29.4, the representation $\Phi$ has 4 irreducible constituents $D_{1}=1, D_{2}, D_{3}, D_{4}$, each with multiplicity 1 . If $f_{i}$ is the degree of $D_{i}$ then $f_{1}=1$ and $\sum_{i=1}^{4} f_{i}=v$.

Let us finally show how the fact that $A$ and $C$ have trace 0 contradicts the integrality of the multiplicities of $\lambda_{1}, \lambda_{2}, \lambda_{3}$. 
In the 3 cases $\lambda_{1}$ appears with multiplicity 1. Let $f$ denote the multiplicity of $\lambda_{2}$; then $v-f-1$ is the multiplicity of $\lambda_{3}$. This leads to

$$
\begin{aligned}
& 0=n(n-1)+f(3+\sqrt{8 n+1}) / 2+(n(n+1)-f)(3-\sqrt{8 n+1}) / 2 \text { in case } I, \\
& 0=(n+1)+f \sqrt{n}+(-\sqrt{n})(n(n+1)-f) \quad \text { in case III, } \\
& 0=(n+1)(n-2)+f(-1+\sqrt{8 n-15}) / 2+(n(n+1)-f)(-1-\sqrt{8 n-15}) / 2
\end{aligned}
$$

in case II2.

In any case this contradicts the fact that $n \geqslant 2$ and $f \geqslant 1$ are integers:

In case II1 this is clear.

In case I suppose that a prime $p$ divides $\sqrt{8 n+1}$. Then $p \nmid n$, hence $p / 5 n+1$ and then $p \mid 3 n$, i.e. $p=3$. This implies that $8 n+1=3^{2 i}$ for some $i \geq 2$ and that $n(5 n+1) / \sqrt{8 n+1}=\left(3^{2 i}-1\right)\left(5 \cdot 3^{2 i-1}+1\right) / 8^{2} \cdot 3^{i-1} \notin \mathbb{N}$.

In case II2 suppose that a prime $p$ divides $\sqrt{8 n-15}$. Then $p \in\{17,23\}$ and $\mathrm{p}^{2} X \mathrm{n}+1, \mathrm{p}^{2} X \mathrm{n}-4$. Hence $8 \mathrm{n}-15 \in\left\{17^{2}, 23^{2}, 17^{2} \cdot 23^{2}\right\}$, i.e. $\mathrm{n} \in\{38,68$, 19112\}. Suppose that $n=38$. Since $G_{P_{0}, P_{2}}$ is transitive on $1_{2}-\left\{P_{0}, P_{1}, P_{2}^{Y_{o}}\right\} \quad|G|$ is even. This contradicts the fact that if $n \equiv 2 \bmod 4$, then the full collineation group is of odd order (Hughes [5]). Suppose that $n \in\{68,19112\}$. Then $n$ is not a square and $n^{2}+n+1$ not a prime. Hence, since $\mathrm{G}$ is flag-transitive, $\mathrm{n}$ is a prime power (Higman and Mc Laugh1in [4]) which is absurd.

\section{REFERENCES}

1. Bachmann, 0., On rank 3 projective designs, Mh. Math. 89 (1980), 175-183.

2. Dembowski, P., Finite Geometries, New York, Springer, 1968.

3. Higman, D.G., Finite permutation groups of rank 3, Math. Z. 86 (1964), 145156.

4. Higman, D.G. and J.E. Mc Laughlin, Geometric ABA-groups, I11. J. Math. $\underline{5}$ (1961), 382-397. 
5. Hughes, D.R., Generalized incidence matrices over group algebras, I11. J. Math. 1 (1957), 545-551.

6. Kallaher, M.J., On rank 3 projective planes, Pacif. J. Math. 39 (1971), 207214.

7. Kantor, W.M., Moore geometries and rank 3 groups having $\mu=1$, Quart. J. Math. Oxford (2) 28 (1977), 309-328.

8. Kantor, W.M., Automorphism groups of designs, Math. Z. 109 (1969), 246-252.

9. Wielandt, H., Finite permutation groups, New York, Academic Press, 1964. 


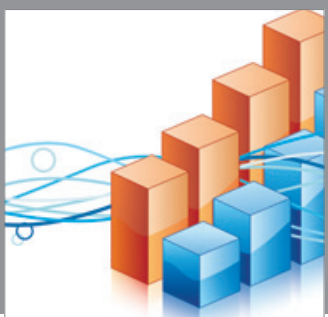

Advances in

Operations Research

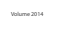

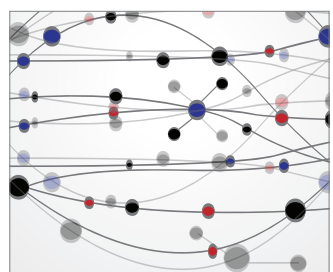

\section{The Scientific} World Journal
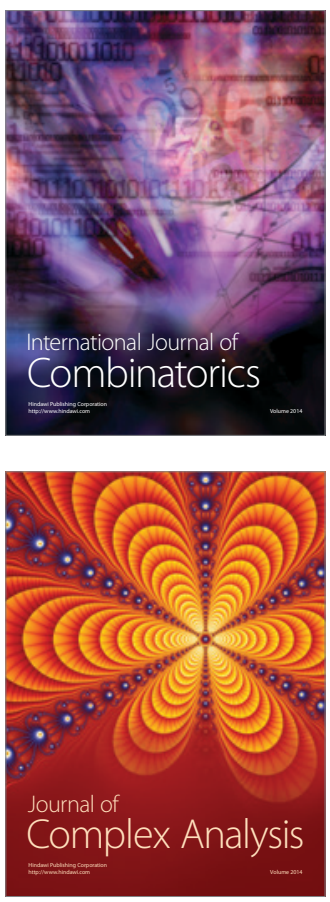

International Journal of

Mathematics and

Mathematical

Sciences
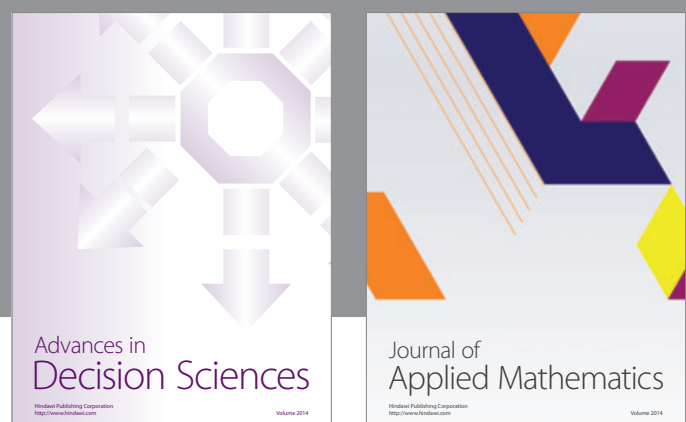

Journal of

Applied Mathematics
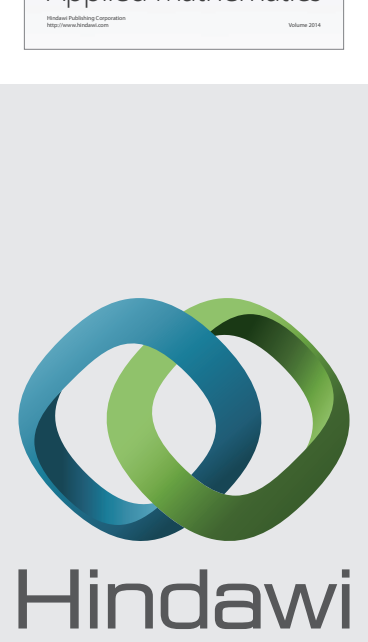

Submit your manuscripts at http://www.hindawi.com
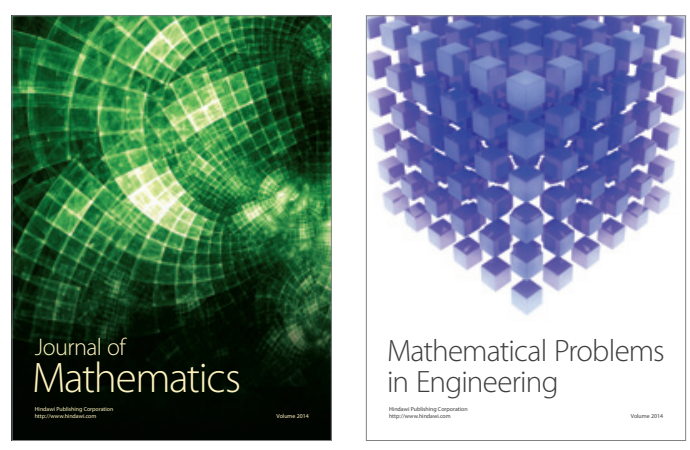

Mathematical Problems in Engineering
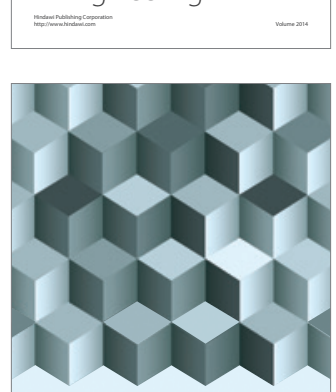

Journal of

Function Spaces
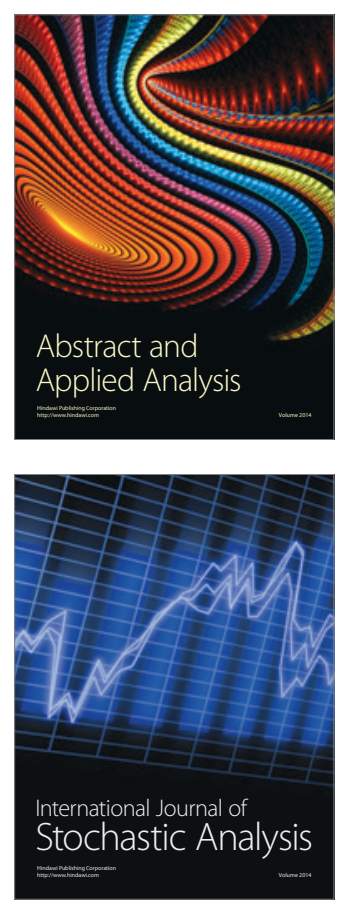

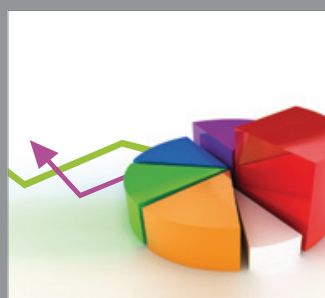

ournal of

Probability and Statistics

Promensencen
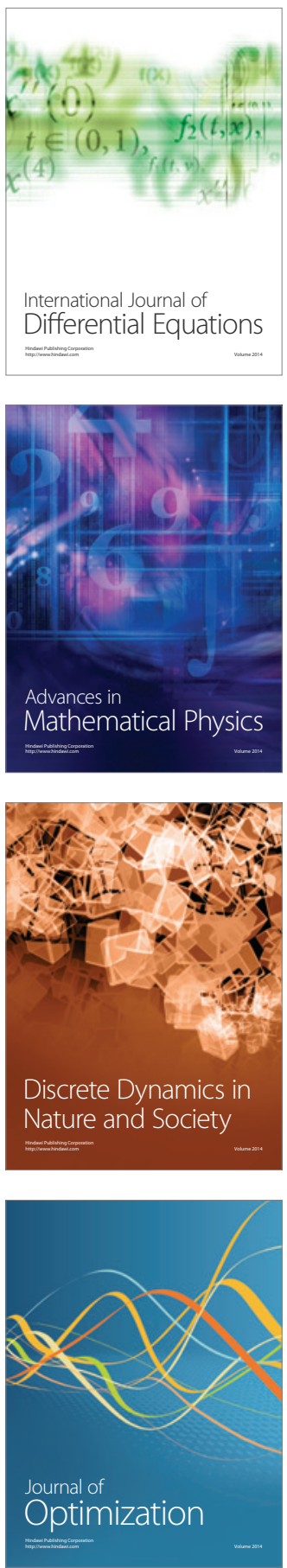